\title{
Temporally Restricted Generics
}

\author{
Yael Greenberg \\ Bar-Ilan University
}

\section{Introduction}

In this paper I present and analyze data which challenges two widely held claims in traditional and more current theories of genericity. The first claim (see e.g. Krifka et al 1995 ) is that genericity and episodicity are mutually exclusive, i.e. that generic sentences, express 'tendentially stable' (in Chierchia's 1995 terminology) properties of their subjects, and not episodic ones. Typical examples which are supposed to support this view are the existential-only interpretation of the bare plural and indefinite singular subjects in (1), and the oddness of the habitual sentences in (2):

a. Dogs are barking now

b. A dog is barking now

\# John walks to school today

In the first part of the paper I show that temporally restricted, or "episodic generics' do, in fact, exist, claim they are indeed generic (and not e.g. "quasi universal" in Condoravdi's 1992 sense) and concentrate on semantic and distributional differences which exist between such sentences with bare plural (BP, henceforth) and indefinite singular (IS, henceforth) subjects. I claim that the underlying difference between such sentences in this construction is a special case of a much more basic pattern of differences between BP and IS subjects, which exist also with more traditional generic constructions, such as sentences with habitual predicates. This challenges a second widely held claim (see e.g. Wilkinson 1991, ter-Meulen 1995, and Chierchia, 1995), namely that when combined with predicates of individuals (i.e. non kind level), sentences with BP subjects (BP sentences, henceforth) as in (3a) should be given the same truth conditions as those with IS subjects (IS sentences, henceforth), as in (3b). Roughly, this is some version of the Chierchian 1995 style representation in (4), namely a tripartite quantificational structure, headed by a universal, unselective, modal operator, Gen (where $\mathrm{C}$ is a context variable taking the contextually relevant situations involving $\mathrm{x}$ ):

a. Birds fly.

b. A bird flies.

$$
\text { Gen s,x [Bird (x) \& C(s,x)] [fly }(\mathrm{s}, \mathrm{x})]
$$

Assigning the same representation to a pair like (3a) and (3b) seems indeed to be justified by the similar, if not equivalent, truth conditions of both sentences. However, in the second part of the paper I point to a number of differences between similar pairs and claim that in general, IS and BP sentences in habitual sentences involve two different sorts of modality, and thus should be represented differently. Roughly the claim is that IS 
sentences express a stronger, "property oriented" kind of nonaccidentalness, whereas BP sentences are also able to express a weaker, "inductive" kind. I formalize this intuition using two kinds of accessibility relation, show how this accounts for the set of differences noted before, and claim that the kind referring/indefinite distinction is the key for explaining this distinction. In the third part of the paper I return to the BP/IS distinction in the temporally restricted construction and show how the property oriented/ inductive distinction can account for it. Section 4 summarizes the paper.

\section{Generically Interpreted BP and IS Subjects in Temporally Modified Sentences}

\subsection{The data}

Unlike the traditional view, BP and IS can both get a generic interpretation as subjects of predicates modified by specific indexical temporal adverbials, as shown in (5) and (6):

a. Italian restaurants are closed today.

b. Americans eat Turkey tonight

a. A Jew is in synagogue tonight.

b. A faithful Catholic is in Church today.

However, in most cases BP are judged as much better in this construction than their IS counterparts. In fact, as seen in (7), the sentences in (6) are exceptional, in that the judgments about most IS sentences in this construction vary between "odd" and "completely unacceptable". In contrast, the minimally contrasting BP sentences are in general perfectly natural with such temporal adverbials, as seen in (8):
a. ?/??/* An Italian restaurant is closed tonight
b. ?/??/* An accountant is very busy this week
c. ?/??/*A lion is very aggressive today
d. ?/??/* A child is especially polite today
e. ?/??/* A Clinton supporter is happy tonight.
a. Italian restaurants are closed tonight
b. Accountants are very busy this week
c. Lions are very aggressive today.
d. Children are especially polite today.
e. Clinton supporters are happy tonight

Despite this general incompatibility of IS sentences with specific temporal idexicals, there are some factors which considerably improve their status. One such factor is the presence of certain kinds of modal expression, like would or should. Notice that it cannot be the mere modality of these expressions which improves the status of the IS sentences, since the presence of e.g. the modal must, under its epistemic interpretation, makes very little or even no improvement at all. All this can be seen in (9): 

a. An Italian restaurant would / ??
b. A lion would / ?? must be very aggressive today.
c. A Catholic should / ??must be nervous today
d. A child should / ?? must be especially polite today.

Another "improving factor" is the addition of modifying "normative" adjectives. For example, the sentences in (10), are much better than those in (7):
a. A decent Italian restaurant is closed today.
b. A true Clinton supporter is happy tonight
c. A decent accountant is busy this week
d. A well-behaved child is especially polite today.

The last "improving factor" for the IS sentences is context. Most informants find the IS sentences better when uttered with supporting context, instead of out of the blue. However, not any context can help here. Specifically, the IS get better in what I will call a "predictional" scenario, but not in an "inductive" scenario. In contrast, BP sentences can appear easily in both kinds of scenario ${ }^{2}$. The following examples illustrate this:

(11) The inductive Italian restaurants scenario: John and Mary plan to meet in town and eat in an Italian restaurant. John arrives in town before Mary, and while walking down the street, he sees that "Mamma Mia" is closed. He then goes to "Little Italy", and then to "Fonta Bella", but they are also closed. Two more Italian restaurants he tries are also closed. When Mary arrives and meets him, John says:

(a) Look, Italian restaurants are closed tonight. Let's go to an Indian restaurant

(b) ??/* Look, an Italian restaurant is closed tonight. Let's go to an Indian restaurant

(12) The predictional scenario: Me and my friend decide to eat tonight in an Italian restaurant, but an hour later my friend calls me and says "We better go eat in an Indian restaurant tonight. I just remembered that its Italy's independence day,

(a) Italian restaurants are closed tonight

(b) ? an Italian restaurant is closed tonight

\subsection{Real generic, or 'quasi-universal'?}

Before turning to account for the rather surprising existence of 'episodic generic' sentences as in (5-12) above, and to explain the differences between the BP and IS sentences, we need to make sure that these subjects are really interpreted generically. Indeed, Condoravdi (1992) examines sentences with BP subjects, similar to those in (5) and (6), as in (13), and claims that despite the 'quasi-universal' reading of the subject, it is not generic in any obvious way:

(13) In 1987 a ghost haunted the campus. Students were aware of the danger. 
Condoravdi claims that unlike classic generic sentences (e.g. A lion has a bushy $\underline{\text { tail }}$ ), sentences like (13) do not express any regularity, are contextually restricted (to students in this campus only), and have existential presuppositions (for the students in the campus). All these properties are also shared in some cases, by the temporally modified sentences in (5) and (6). If, then, real generic sentences never have these properties, as Condoravdi claims, (specifically the second and third properties), then what I called before "temporally restricted generic" may not be generic at all.

Notice, however, that unlike Krifka's (1987 claim, which Condoravdi follows here, generic sentences can be contextually restricted, e.g. the subjects in (14) (noted by Fred Landman, p.c.) can be easily understood as professors in this university:

(14) There are students and professors in this university. Professors wear / A professor wears a tie

In addition, although many generic sentences indeed do not presuppose the existence of actual instances, such a presupposition is not a necessary indication of nongenericity. In fact, most past-tense generics have this property:

a. In the middle ages, astronauts/an astronauts earned lots of money

b. \# But in fact there were no astronauts in the middle ages

The infelicity of (15b) indicates that (15a), which is a clear generic sentence, presupposes the existence of astronauts in the middle ages. Thus, besides the episodicity of (13), and for the same purpose (5) and (6), which is indeed surprising (and is the target of examination of this paper), there is no indication that such sentences are significantly different from classic generics.

Moreover, we have positive indications that sentences as in (5), (6), and (13) are in fact generic. In particular, they share with classic generic sentences three properties which have been identified as central for the characterization of genericity. Specifically, like classic generics, (5), (6), and (13) all allow for exceptions. I.e. they are true even if not all members of the set denoted by the subject have the predicated property. Secondly, they all support counterfactuals. For example, both (5a) and (6a)) support (16):

(16) If this were an Italian restaurant it would be closed tonight (pointing at an Indian restaurant)

Thirdly, like other classic generics, these sentences all obey Cohen's (1995) "homogeneity constraint" illustrated with the following example: Imagine a situation where $75 \%$ of the Chinese restaurants are in Chinatown, and all of them are closed tonight, while all other Chinese restaurants in the rest of the town are open. This kind of situation easily supports the truth of (17a), but clearly not (17b):

(17) a. Most Italian restaurants are closed tonight

b. Italian restaurants are / ??An Italian restaurant is closed tonight 
The conclusion is, then, that both Condoravdi's original examples, as well the temporally modified sentences in (5-6), behave like real generic sentences in several important respects. It is time, then, to examine these sentences more closely.

\subsection{A descriptive generalization: Two readings of the temporal modifier.}

Let me turn back now, to the differences between IS and BP sentences when indexical temporal adverbials are present. Remember that the basic pattern was that BP sentences are perfectly natural in this construction, whereas in most cases, such IS sentences are infectious, at least to some extent, unless some specific "improving factors" are present. Understanding this basic pattern depends on distinguishing two readings of the temporal indexical adverbials like tonight. Such adverbials have their natural, default interpretation, where they denote simply temporal locations which are part of the night, day, week, etc. in which the utterance is made. This is the reading we most naturally get in nongeneric sentences like Mary at the movies tonight and which is written more formally in (18). In this case the temporal indexical specifies that the temporal location of the existentially closed situation variable is part of the night of utterance ${ }^{3}$.

$\exists \mathrm{s}$ [at-the-movies (s) \& $\mathrm{Ag}(\mathrm{s})=$ Mary \& loc. $(\mathrm{s}) \leq$ night of the utterance]

However, such adverbials have another interpretation, as denoting values to temporal functions such as "Italy's independence day", "John's birthday", "Sunday", etc. In this case, the indexical carries with it, besides the temporal location it denotes, some property or qualification of the temporal location. In simple, "out of the blue" sentences we normally don't get this second reading. But there are some linguistic and pragmatic factors which trigger it. For example, pieces of discourse as in (19) and (20) suggests that the modals would, should and the deontic must are necessarily such triggering factors, but the epistemic modal must is also compatible with the "temporal location only", reading:

(19) a. John: Mary would / should / must behave very politely to her boss today. Bill: Why ? what's special about today?

b'. John: It's his birthday / It's her first day at this job / It's Friday and he is always nervous on Fridays / etc. .

b'. John: \# I have no idea. As far as I know this is just a regular day.

(20) a. John: Mary must have behaved very politely to her boss today. Bill: Why ? what was special about today?

b'. John: It's his birthday / It's her first day at this job / It's Friday and he is always nervous on Fridays / etc..

b" John: I have no idea. As far as I know this is just a regular day. (I just saw him smiling a lot to her, so I figured that out.)

In both (19) and (20) there are two possible answers to Bill's question "What's special about today?". (b') gives some possible properties of the temporal indexical, i.e. 
specifies the function that the indexical is a value of, whereas (b') asserts that the speaker has no such property or function in mind. This last answer, however, is infelicitous when an overt deontic modal is present in the sentence. This suggests that with such modals the speaker has to have in mind, and the listener has to accommodate, some property associated with the temporal location. As seen from the felicity of (20b'), this is not necessarily the case with the epistemic must ${ }^{4}$.

With this distinction in mind we can now make a descriptive generalization:

a. IS sentences are compatible only with the "value to function" reading of temporal indexical adverbials.

b. BP sentences are compatible with both the "temporal location" and the "value to function" readings of temporal indexical adverbials.

The generalization in (21) can account now for the full range of data described in section 1.1. BP sentences are perfectly natural in this construction since they are compatible with the default reading of the temporal indexical, whereas the general infelicity of IS sentences results from their compatibility only with a nondefault reading, which is triggered in specific cases only. Indeed, all the cases where the status of such sentences improves are those where the "value to function" reading is triggered. For example, the contrast between the felicitous versions of (9), with would and should, and the infelicitous ones with epistemic must, results from the fact seen above, that the former modals "force" the functional reading of the temporal indexical, whereas the latter is also compatible with the "temporal location" reading. Presumably, in the latter version of (9), when no other triggering factor is present, the default reading of the temporal is again preferred, thus making the IS sentences infelicitous. The addition of normative adjectives, as in (10), introduces implicit deontic modality into the sentences, which again triggers the functional reading of the temporal. In (12) the functional reading is triggered simply by an explicit reference to the temporal function (e.g. Italy's independence day). Finally, in (6), it is world knowledge which make the functional reading (e.g. Thanksgiving, A Sunday, etc.) available.

It seems, then, that the generalization in (21) above is along the right lines. The next task, of course, is to explain it. The present theories of genericity do not seem to help here. For example, assuming the common view for the representation of IS sentences, i.e. as bound by an unselective universal modal operator (as in (4) above) cannot explain why it is that IS sentences are incompatible with the "temporal location" reading of the indexical. This is because there is nothing in the nature of Gen, as defined by these theories, which will make it necessarily incompatible with this reading, and will rule out a representation such as (22) for \# An Italian restaurant is closed tonight:

(22) Gen $\mathrm{s}, \mathrm{x}[\operatorname{IR}(\mathrm{x}) \& \mathrm{C}(\mathrm{s}, \mathrm{x}) \&$ loc. $(\mathrm{s}) \leq$ night of the utterance] [closed $(\mathrm{s}, \mathrm{x})]$

"Every contextually relevant situation whose temporal location is part of the night of utterance, involving any Italian restaurant $(\mathrm{x})$ in all accessible, close enough worlds, is a situation where $\mathrm{x}$ is closed." 
That is, the "temporal location" reading of the temporal indexical can go under the scope of a modal operator, and be interpreted as "tonight in all accessible worlds". This claim is independently claimed in L.T.F. Gamut 1991 w.r.t. temporal indexicals in the antecedents of counterfactuals as If I hadn't gone to the party tonight, I wouldn't have met you. Here too the temporal axis stays constant along the variation across worlds.

However, as the data shows, this reading is impossible for the IS sentences, and we need to have additional information, or property about the temporal location. In addition, the dominant approach, which assigns BP and IS sentences (with non kind level predicates) the same representation (see again (3-4) above), cannot explain the difference between them. Problems arise even if we choose to exploit the D/I genericity distinction and represent the BP sentences as "Proper kind predication" as in (23):

closed tonight (ITALIAN RESTAURANTS) (capitals indicate kinds).

The problem in this representation is that it cannot explain the "classic generic" properties of the temporally restricted BP sentences, as noted in section 1.2 above, and especially not their counterfactual supporting property, i.e. the fact that despite their episodicity, they express some sort of nonaccidental generalizations. This is clearly not a necessary property of proper kind predication in general, as can be seen from the accidental nature of the proper kind predication sentences in (24) and (25):

a. Rats reached Australia in $\mathbf{1 7 8 6}$

b. Dodos are extinct.

To conclude this section, we saw that the generalization in (21), in terms of the compatibility with two possible readings of the temporal indexical, can account for the pattern of differences between BP and IS sentences described in section 1.1. However, trying to explain this generalization in terms of the present theories of genericity does not work. My claim is that the generalization in (21) is, in fact, a special case of a much more basic and wide difference between BP and IS sentences, which can be understood only by looking at a wider set of data. In the next section I examine the differences between BP and IS sentences with habitual predicates, and end up with a finer grained semantics for the generic operator. In the last section I will show that the habitual data is strikingly similar to the temporally restricted data, and use the former to account for the latter.

\section{Differences between BP and IS sentences with habitual predicates.}

\subsection{The old intuition and the new data}

There has been several observations in the past about semantic and distributional differences between BP and IS sentences with predicates of individuals. The observations I want to concentrate on are those made by Burton Roberts 1977 , Declark 1986 and Krifka (1987), which said roughly that IS sentences express "analytic", "definitional" or "normative" statements, and predicate "inherent" properties of their IS subjects, whereas 
BP sentences express more "descriptive", "inductive" statements, and their subjects combine with more "accidental" properties. Burton Roberts, for example, claims that in a minimally contrasting pair as in (26) this difference shows up very clearly:

(26) a. A gentleman opens the door for ladies.

b. Gentlemen open the door for ladies.

I believe these observations are basically right, but on the intuitive level they stand now they are not very helpful. It is not clear, for example, what the exact meaning of "inherent" and "accidental" properties is, and moreover, assuming that habitual BP sentences support counterfactuals and express "nonaccidental statements", what it mean for them to predicate "accidental" properties of their subjects. In addition, there is quite a bit of data which cannot be accounted for by the old intuition. The main problem is that, many if not most minimally contrasting pairs of BP and IS sentences do not seem to differ semantically at all (see again (3) above). In addition, as correctly shown by Cohen 1997, IS subjects can combine also with highly contingent, noninherent, properties, as in e.g. $A$ carpenter in this town earns lots of money.

At this point I want to add some new observations about the BP/IS distinction, which the old intuition cannot explain, but which eventually will help us to refine it.

Notice firstly, that when the subjects denote an extremely "unnatural class", informants report a prominent existential reading of the IS subject, despite the habitual predicate, which is supposed to ensure a generic interpretation only. In contrast, the BP subject is interpreted generically only (or most prominently) as expected:

(27) A Norwegian student whose name ends with ' $s$ ' or ' $j$ ' wears green thick socks.

(28) Norwegian students whose name end with 's' or ' $j$ ' wear green thick socks.

(29) A tall, left handed, brown haired Neurologist in "Hadasa" earns more than $50000 \$$ a year.

(30) Tall, left handed, brown haired Neurologists in "Hadasa" earn more than $50000 \$$ a year.

We can find the same pattern also with relatively "natural classes" subjects, combine with extremely "unconnected" properties, as seen in the following pairs

(31) a. A carpenter in Amherst earns very little.

b. A carpenter in Amherst gives all his sons names ending with ' $a$ ' or ' $g$ '.

(32) a. Carpenters in Amherst earn very little.

b. Carpenters in Amherst give all their sons names ending with ' $a$ ' or ' $g$ '.

The IS sentence in $(31 \mathrm{~b})$ gets a much more prominent existential reading than the one in (31a), which has a much more readily available generic reading. The BP subjects get a prominent generic reading in both (32a) and (32b).

Notice, though, that the generic reading is not completely blocked for the IS sentences in (27), (29) and (31b), and as a speaker I can choose to express a generalization using either the IS or the BP forms in (27)-(32). But even here, for the 
speakers, there are restrictions on the use of IS sentences. There are some situations which will license the use of BP sentences, but not of IS one. One such kind of situations is pure inductive reasoning. Thus, if I walk through the campus and meet ten Norwegian students whose names end with ' $s$ ' or ' $j$ ', all of whom wear thick green socks, I can use the BP sentence in (28) very naturally to express a surprising generalization about such students. But this information does not seem enough to license the IS sentence in (27) (on its generic reading). It is as though we need something more than the mere observation of $\mathrm{x} 1, \mathrm{x} 2, \mathrm{x} 3 \ldots \mathrm{xn}$ instances having the predicated property in order to say such an IS sentence.

A clue about what this "something" is can be found in the fact that a speaker can use such IS sentences generically, and listeners report a much more prominent generic reading, when the IS sentences are not uttered out of the blue, as above, and not against pure inductive contexts, but as continuations of the following:

(27 $)$ You know, there are very interesting traditions in Norway, concerning the connection between the name, profession and clothing, for example (a Norwegian student...)

(29) The new "Hadasa" manager has some very funny paying criteria, for example (A left handed......)

(31b ') Joshua Greenberg was a famous and admired carpenter in Amherst a hundred years ago. From that time until now (a carpenter in Amherst...)

\subsection{A new intuition: "property oriented" vs. "Inductive" generalizations.}

Here is what I intuitively think is going on with IS and BP sentences. We have two possible kinds of modalized generalization encoded in natural language, which I will call "property oriented" and "inductive". IS sentences can express only property oriented generalizations. This means that they can only be asserted if the speaker has in mind, and the listener can accommodate, some specific (at least to some extent) property associated with the set denoted by the IS subject, in virtue of which, or because of which, every member of the set has the predicated property. For example, A boy doesn't cry will be true, roughly, if there is some property we associate with the set of boys - a genetic property, or a social norm property, e.g. being tough, in virtue of which every member of this set will not cry (in all contextually relevant situations). Notice that many times a speaker can assert an IS sentence even if he or she only knows the "sort of property" which causes the predicated property. So, for example, I can assert $A$ pentium computer works very fast, as a computer expert, having in mind this sophisticated processor, DH200, installed in those computers and making them work fast, but also as a layperson, meaning simply that "in virtue of their mechanical properties, pentium computers work very fast".

Unlike IS sentences, BP sentences can express both "property oriented generalizations", but also a second, inductive, kind of generalization. the latter assert, on the basis of several actual instances of the set having the predicated property, that "there is some pattern here", i.e. that this cannot be accidental and restricted to these instances only, i.e. that any other member of the set will have the predicated property (in all 
contextually relevant situations). In the case of e.g. Boys don't cry, we can imagine an alien from Mars visiting our planet and watching the behavior of children. At some point, after seeing the behavior of several boys in several "tear inducing situations", he decides that there is some pattern here, and that the next boy in such a situation will not cry either. Crucially, at this stage he does not know or care, and his statement does not involve, not even in an implicit manner, the property which makes boys not to cry. All he claims is that "this is not accidental", i.e. not limited to the observed boys only.

The intuitive distinction just described reminds of, and was inspired by Carlson's (1995) distinction between the inductive and the "rules and regulations" approaches to genericity. Carlson, however claims that we should choose one of these approaches to capture the variety of genericity phenomena. My claim is that natural language encodes both kinds of generalization.

\subsection{Two accessibility relations.}

In order to make the intuitive distinction above more precise we have to find some component in the formal representation which can express it. My suggestion is to let the modal base (in the sense of Kratzer 1981) do the work. This is reasonable, among other things because, as seen in section 2.1 above, sometimes the expected generic reading of an IS subject appears only when the sentence is uttered against some context, instead of out of the blue (cf. again (27)-(27')). This suggests that the context prevents the need of accommodation of some sort, which in the case of some IS sentence is more difficult to achieve than with BP ones. It is independently argued (e.g. by Kratzer 1981) that in "out of the blue" modalized sentences the modal base must be accommodated.

Here is, then, a possible representation of the two kinds of generalizations. At this point, both have the same semantic structure, namely they are both tripartite structures headed by a universal quantifier over individual variables, situations and worlds. The difference between them now is only the accessibility relation, (i.e. modal base):

Property oriented generalizations are true in a world $w$ iff $\forall \mathrm{x}, \mathrm{s}, \mathrm{w}^{\prime}[\operatorname{Subj}(\mathrm{x}) \& \mathrm{R}(<\mathrm{w}, \mathrm{x}>$, w') \& $\mathrm{C}(\mathrm{s}, \mathrm{x})$ ] [Pred $(\mathrm{s}, \mathrm{x})$ ] Where $\mathrm{R}(<\mathrm{w}, \mathrm{X}>, \mathrm{w}$ ') gives the set of world accessible from $\mathrm{w}$ w.r.t. some property, or second order property of $\mathrm{x}$, which is contextually chosen from the cluster of properties associated with $\mathrm{x}$ in $\mathrm{w}$.

That is, we assume that we can associate with every set denoted by a common noun, or a complex NP, a cluster of properties that every member of the set has. Crucially, not all these properties should be in fact true of every member of the set in the actual world. So, for example, in our world I associate with the set of boys the properties of being a male, being under sixteen years of age, being tough, loving sports etc. The first property is true of all boys in the actual world (and in fact in all worlds where English behaves as in the actual world), the second one is true of all boys in the worlds where, more or less, standards of modern western culture hold, the third and fourth properties are true in all worlds where some specific social standard holds (it may be that in the actual 
world no boy is in fact tough). An important point is that this cluster of properties I am talking about need not include only stereotypical properties, of well established classes. Thus, I may associate with the set carpenter in Amherst properties that only I have in mind, in virtue of some information that only I have access to, e.g. required to pay high taxes.

We may also associate with a set a cluster of second order properties (what I called before "sorts of properties"). So, for example, we tend to think about computers as having mechanical properties, even without being computer experts and knowing what these properties are. Similarly, we usually associate with boys social standards in general, with animals and plants biological / genetic properties, with professions like carpenters, lawyers and doctors economical and legal properties, etc. The cluster of second order properties we associate with the set $\mathrm{Q}$ in $\mathrm{w}$, then, is the set of sets of first order properties, such that some (possibly unknown) first order properties in this set are true of every member of $\mathrm{Q}$ in the worlds accessible from $\mathrm{w}$.

Now the cluster of properties associated with $Q$ in $w$ can be defined as the union of the set of properties and second order properties that every member of $Q$ has in all the sets of worlds which are epistemically, deontically, socially, streotypically, etc. accessible from w. More formally (following the spirit of Kratzer 1981) :

The cluster of properties associated with a set $Q$ in $w$ :

$\lambda \mathrm{P} . \forall \mathrm{x}[\mathrm{Q}(\mathrm{x})][\mathrm{P}(\mathrm{x})] \mathrm{w} \cup \lambda \mathrm{P} . \exists \mathrm{P}[\mathrm{P}(\mathrm{P})][\forall \mathrm{x}[\mathrm{Q}(\mathrm{x})][\mathrm{P}(\mathrm{x})]] \mathrm{w}^{\prime}$

Where $\mathrm{w}^{\prime} \in \cap\left(f(\mathrm{w}) \cup f(\mathrm{w}) \cup f^{\prime}(\mathrm{w}) \cup f^{\prime \prime}(\mathrm{w}) \ldots.\right)$

(where $\mathrm{Q}$ and $\mathrm{P}$ are of type $<\mathrm{e}, \mathrm{t}>, \mathrm{P}$ is of type $<<\mathrm{e}, \mathrm{t}>, \mathrm{t},>$ and where $f, f^{\prime}, f^{\prime}, f^{\prime}$, ,

are various epistemic, deontic, stereotypical etc. conversational backgrounds).

Now, the accessibility relation $R\left(\left\langle w^{\prime}, x\right\rangle, w^{\prime}\right)$ in (33) (inspired by Brennan's 1993 idea of "accessibility relation keyed to properties of individuals) gives us the set of all worlds where every member of Q (the subject set) has a first order property, or some first order property in the set of properties, which is contextually chosen out of this cluster:

$\mathrm{R}(<\mathrm{x}, \mathrm{w}>$, w') holds iff one of the following holds:

(i) $\mathrm{w} \in \forall \mathrm{x}[\mathrm{Q}(\mathrm{x})][\mathrm{P}(\mathrm{x})]$ (for a contextually chosen property $\mathrm{P}$ )

(ii) $\mathrm{w} \in \exists \mathrm{P}[\mathrm{P}(\mathrm{P}) \& \forall \mathrm{x}[\mathrm{Q}(\mathrm{x})][\mathrm{P}(\mathrm{x})]$. (for a contextually chosen second order property $\mathrm{P}$ )

An important point is that one of the main factors influencing the choice of the specific property out of the cluster, is the predicated property in the sentence. The chosen property (or second order property) cannot be any arbitrary property in the cluster, but should be able to stand in a specific relationship to the predicated property (denoted by the VP), which I intuitively call "in virtue of" or "causal" relationship. Thus, for example, reasonably chosen properties in the case of $A$ Penguin book is difficult to read can be the size of the letters, the color of the pages, or the types of topics dealt with in these books, but not e.g. the color of the cover, although the latter is also one of the possible (and even stereotypical) properties in the cluster associated with "A Penguin book". At this point I will not define formally the "in virtue" or "causal" relationship between the chosen and 
predicated property, and continue to talk about this "in virtue of" requirement on the contextually chosen property in an intuitive manner (a promising direction to take here is Lewis's 1986 discussion of causality in terms of counterfactual relation).

The following gives a concrete example of property oriented generalizations:

A pentium computer works very fast is true in $\mathrm{w}$ iff $\forall \mathrm{x}, \mathrm{s}, \mathrm{w}^{\prime}$ [ pentium computer $(\mathrm{x}) \& \mathrm{R}\left(<\mathrm{w}, \mathrm{x}>, \mathrm{w}^{\prime}\right) \& \mathrm{C}(\mathrm{s}, \mathrm{x})$ ] [works very fast $(\mathrm{s}, \mathrm{x})$ ]. I.e. iff every contextually relevant situation (e.g. a one where the computer works) involving every pentium computer $(\mathrm{x})$ in all worlds where every pentium computer has a DX-200 processor (or alternatively, has some mechanical first order property) is a situation where such an $\mathrm{x}$ works very fast.

Let me turn now to the representation of inductive generalizations:

(37) Inductive generalizations are true in a world $w$ iff $\forall \mathrm{x}, \mathrm{s}, \mathrm{w}^{\prime}[\operatorname{Subj}(\mathrm{x}) \& \mathrm{R}$ '(w,w') \& $\mathrm{C}(\mathrm{s}, \mathrm{x})$ ] [Pred $(\mathrm{s}, \mathrm{x})$ ]

Where $\mathrm{R}^{\prime}\left(\mathrm{w}, \mathrm{w}^{\prime}\right)$ gives the set of worlds which are just like w, except for what is needed in order to allow for the existence of more instances of Subj (x) and $\mathrm{C}(\mathrm{s}, \mathrm{x})$, and which are closest to an ideal world w' determined by an ordering source $g$, where no surprises exist w.r.t. $\operatorname{Subj(x)}$ in such situations.

Notice that in this case we have both a new modal base, R', and an ordering source (in the sense of Kratzer 1981). The latter defines an ideal world where e.g. no surprises occur w.r.t. boys in tear inducing situations. This is very similar to Dowty's 1979 inertia worlds or Chierchia's 1995 "stereotypical cases". The purpose of this ordering source is to ensure that surprising situations involving boys, caused by external factors, and in which boys don't cry, will count as legitimate exceptions and will not falsify the generalization. following:

For example, the inductive reading of Boys don't cry will be represented as in the

Boys don't cry is true in w iff $\forall \mathrm{x}, \mathrm{s}, \mathrm{w}^{\prime}$ [ $\operatorname{Boys}(\mathrm{x}) \& \mathrm{R}$ '(w,w') \& $\left.\mathrm{C}(\mathrm{s}, \mathrm{x})\right][[\neg$ cry $(\mathrm{s}, \mathrm{x})]$, i.e. iff for every boy, and every tear inducing situation involving a boy, in all worlds which are just like w, except for what is needed in order to allow for the existence of more boys and more tear inducing situations, and which are closest to an ideal world where no surprises exist, w.r.t. boys in tear inducing situations, every boy will not cry in such a tear inducing situation.

\subsection{Accounting for the data}

Let me repeat the claim I made in the beginning of section 2.2 :

(39) a IS sentences can express property oriented generalizations only.

b. BP sentences can express both property oriented and inductive generalizations 
Having defined the two kinds of generalization using two kinds of accessibility relations, we can now turn to account for the data described in section 2.1. Firstly, the fact that the IS sentences are only restricted to accessibility relations which are sensitive to clusters of properties we associate with the subject set, explains the fact that when the IS expresses "an extremely unnatural class", and when the sentence is uttered out of the blue, it gets a prominent existential reading (see again $(27,29)$ above). This is because intuitively, such classes (like $A$ Norwegian student whose name ends with ' $s$ ' or ' $g$ ') are those which our common ground knowledge does not associate any property with (besides the property of belonging to this set itself). In such a case, for the typical listener, no property or second order property will be contextually chosen out of the cluster of properties, because this cluster simply doesn't exist. Thus the accessibility relation cannot be defined, the generic reading fails, and the listener turns to the available existential reading.

Let me turn now to explain the contrast between $(31 a-b)$, repeated here:

a. A carpenter in Amherst earns very little.

b. A carpenter in Amherst gives all his sons names ending with ' $a$ ' or ' $g$ '.

The subject of $(3 \mathrm{lb})$ gets a prominent existential reading (again, only out of the blue), but unlike what happens with (27), this cannot be due to the subject, because (31a), with exactly the same subject, gets a generic reading naturally. Instead, the reason for the contrast lies in what I intuitively called above the "in virtue of" requirement on the contextually chosen property. Out of the blue, listeners can easily accommodate a second order property which causes every carpenter in Amherst to earn very little, e.g. some economical or legal property of this profession. However, Given the standards of western culture, it is much harder to accommodate a property in virtue of which every such carpenter will give his sons names ending with certain letters.

These difficulties in accommodation are resolved immediately if the "in virtue of properties" are explicitly referred to by the speaker. This is why the generic reading of the "problematic" cases is much more easily available in (27') (29') and (31b') above.

As shown in section 2.1., BP subjects are unlike IS ones in being naturally interpreted as generic even when denoting extremely unnatural classes, or combining with "unconnected" properties. The reason for this contrast is that, as claimed above, BP sentences can also express inductive generalizations, whose accessibility relation is not sensitive at all to the associated cluster of properties. Thus, with such generalizations, the listener need not accommodate any "in virtue of" property. The assertion that "there is a pattern here", or that any situation involving any $x$ would hold in any close enough world where we find more Xs and situations, can thus apply even to the most unnatural or bizarre sets, and to any "unconnected properties". On the other hand, inductive reasoning contexts (where I observe $\mathrm{x} 1, \mathrm{x} 2, \mathrm{x} 3 \ldots \mathrm{xn}$ instances having the predicated property) cannot trigger the generic interpretation of the "problematic" IS sentences above, because crucially such contexts don't supply any information about possible relevant ("in virtue of') properties of e.g. Norwegian students whose names end with ' $s$ ' or ' $g$ ' . 


\subsection{Referentiality enters the picture}

Before turning to account for the temporally restricted data using the two accessibility relations, we have to relate to an obvious and important question. If IS and BP sentences are given roughly the same semantic structure, what causes the difference between them?

A possible way to answer this is to exploit the kind referring / indefinite distinction (as already suggested by Krifka 1987 for the "old intuition"). The main support for this direction comes from the behavior of habituals with proper name subjects, as in (39):

a. John walks to school

b. Mary handles the mail from Antarctica (Carlson 1977)

Just like BP sentences, sentences such as (39a) and (39b) can express both kinds of generalization. As pointed out by Carlson 1995, the most natural reading of (39a) is the "weak descriptive" one, or the "inductive", in our terms, which is based on watching John's walking to school for e.g. ten days in a row. But (39a) can also express a property oriented generalization, asserting, e.g. that John walks to school in virtue of not having a car, or living close to school (imagine John's mother saying (39a) to a neighbor, without even seeing John's walking to school). Similarly, (39b) can assert that Mary handles the mail from Antarctica in virtue of, e.g. working in this post office (in this case, as pointed out by Carlson 1995, it can be true with no actual handling Antarctica mail situations). It can, however, be naturally uttered also after seeing Mary handling this mail for several days, thus expressing an inductive generalization.

The situation can be schematically described now in (40):

a. Sentences with IS subjects: property oriented generalizations only.

b. Sentences with BP subjects

Sentences with proper name subjects:

Both property oriented and inductive generalizations.

The pattern in (40) can be explained as follows. Unlike the IS subjects, which necessarily denote properties, BP subjects are independently known to be potentially kind referring, i.e. potentially referential ${ }^{6}$. This is what clusters together sentences with BP and proper name subjects, as opposed to IS sentences, and what seems to influence the difference in compatibility with the two kinds of generalizations. My suggestion (which is still intuitive and needs further independent support), is that unlike set or property expressions, with which we necessarily associate clusters of properties, as described above, individuals, denoted by referential expressions, are only potentially associated with such clusters. Thus, hearing the name John, I can either have in mind the individual referred to by John, or, in addition, a cluster of properties I associate with this individual, like being male, having brown hair, living next door, not having a car, etc. Similarly, kinds, denoted by BPs, can be either conceived as some sort of individual, e.g. a plural, collective individual as in Chierchia 1996 and Dobrovie-Sorin and Laca 1996, or as this individual associated with various possible properties (which can be the same properties I associate with the corresponding set denoted by an IS). In both cases, the proper name and the BP 
subjects are represented as referential expressions, but when they appear in the scope of a generic operator, they are compatible with two different accessibility relations, a property oriented one, which is sensitive to the cluster of properties, and an inductive one, which is blind to it. In contrast, when this modal operator binds a restricted $\mathrm{x}$ variable, i.e. a property expression, the associated cluster of properties necessarily enters the picture, and forces the property oriented accessibility relation.

If this line is correct, it should be manifested, of course, in different structures for IS and BP sentences. I will schematically give here two possible structures for BP sentences, both of which are tripartite structures headed by a modal operator (over worlds). The first possibility is to let this operator bind also minimal situations involving realizations, as in (41) (in the spirit of e.g. De Swart 1994), whereas the generic operator binding IS sentences will directly bind an $x$ variable as well (As in (33) above) ${ }^{7}$ :

$$
\begin{gathered}
\text { Boys don't cry: } \forall \mathrm{w}^{\prime}, \mathrm{s}[ \\
{\left[\exists \mathrm{x} \mathrm{x} \leq \mathrm{BOYS} \& \mathrm{C}(\mathrm{s}, \mathrm{x}) \& \mathrm{R}{ }^{\prime}\left(\mathrm{w}, \mathrm{w}{ }^{\prime}\right)\right]} \\
{[\exists \mathrm{x} \mathrm{x} \leq \mathrm{BOYS} \& \neg \mathrm{cry}(\mathrm{s}, \mathrm{x})]}
\end{gathered}
$$

The second possibility is to let the BP sentences express modalized proper kind predication structures, over the collectivity, or totality of instantiations (in the spirit of e.g. Chierchia 1996). I.e. to let the generic operator bind only worlds, as in $(42)^{8}$ :

$$
\forall \mathrm{w}^{\prime}\left[\mathrm{R}^{\prime}\left(\mathrm{w}, \mathrm{w}^{\prime}\right)\right][\neg \mathrm{cry}(\mathrm{BOYS})]
$$

\section{Back to temporally restricted generics.}

\subsection{The similarities with habitual sentences}

It is now time to go back to the data discussed in the beginning of this paper. Before turning to account for the differences between BP and IS sentences in this construction, using the two accessibility relations developed above for the habitual construction, I want to point out several similarities between the two sets of data.

The first and most general similarity is that in both constructions the distribution of the IS sentences is more restricted than their BP counterparts, with which "anything goes". Secondly, in both cases, one of the clearest restrictions on the IS sentences is the introduction of explicit inductive reasoning context. Thirdly, in both cases the BP sentences can have a more "accidental", "actual", or "descriptive" flavor than the corresponding IS sentences, which seem to express a stronger and more "definitional" or "normative" type of lawlikeness. Finally, in both constructions, the status of the infelicitous IS sentences is improved considerably when uttered against some context, instead of out of the blue (cf. again (7a) with (12) and (27) with (27')).

These similarities suggest indeed that the two sets of differences between BP and IS sentences are part of the same phenomenon and should be accounted for in the same way. In the next section I finally turn to account for the temporally restricted data using the distinction I found in the habitual construction. 


\subsection{Explaining the descriptive generalization}

The variety of differences between BP and IS in temporally restricted sentences are due to one underlying distinction, summarized in (21) and repeated here:

a. IS sentences are compatible only with the "value to function" reading of temporal indexical adverbials

b. BP sentences are compatible with both the "temporal location" and the "value to function" readings of temporal indexical adverbials.

The reason for the restriction on the IS sentences seems now to be the following. In order for an IS sentence to be compatible with a temporal indexical in the "temporal location" reading, it should have the following truth conditions:

\#An Italian restaurant is closed tonight is true in $\mathrm{w}$ iff $\forall \mathrm{x}, \mathrm{s}, \mathrm{w}$ ' [IR (x) \& temporal loc. (s) $\leq$ night of utterance \& $\mathrm{C}(\mathrm{s}, \mathrm{x}) \&$ $\left.\mathrm{R}\left(<\mathrm{x}, \mathrm{w}>, \mathrm{w}^{\prime}\right)\right]$ [closed $((\mathrm{s}, \mathrm{x})$ ]

The problem seems to lie in the "in virtue of" requirement on the contextually chosen property, which requires that the relevant property be one which in principle can cause the predicated property. In this case, however, the predicated property is "being closed in a situation whose temporal location is part of the night of utterance". But, what properties associated with the set of Italian restaurants can be responsible, or cause such a property? Intuitively, there are simply no such possible appropriate properties in the cluster. This is because the properties we associate with sets are inherently independent of when John, Mary, or I utter a specific sentence. (In this sense, the problem with this reading is similar to the problem with the "unconnected properties" of (31b) above).

As shown in section 1, the status of the IS sentences becomes better only if there are linguistic or pragmatic factors which trigger the interpretation of the temporal indexical (e.g. tonight) as having some properties, which are independent of the time of utterance. In the case of An Italian restaurant is closed tonight, the predicated property can be now something like "being closed on Italy's independence day, which happens to be tonight". Now we can reasonably find a property in the cluster of properties we associate with Italian restaurants, which is causally connected to Italy's independence day (or a similar date), such as being owned by people who are very patriotic, or who obey traditional, social, or legal standards or rules concerning national holidays, etc.

Unlike the IS sentences, the BP ones are perfectly natural with the default, temporal location reading, of the indexical, because when expressing inductive generalizations, the accessibility relation is not sensitive at all to the cluster of properties associated with the subject set, and thus the problem with the "unconnected properties", which are temporally located at the utterance time, does not rise at all. Instead, in the inductive generalizations the temporal location of the situation, which equals (or part of ) the utterance time, stays constant across the worlds in which there are more members (or realizations) of the set (or kind) denoted by the BP (so, we are talking about "tonight in all 
accessible worlds"). This can be seen in the (44) (alternatively we can use one of the structures suggested in (41) and (42)):

Italian restaurants are closed tonight is true in $\mathrm{w}$, iff

$\forall \mathrm{x}, \mathrm{s}, \mathrm{w}$ ' [IR ( $\mathrm{x}) \&$ temporal loc. (s) $\leq$ night of utterance \& $\mathrm{C}(\mathrm{s}, \mathrm{x}) \& \mathrm{R}$ '(w,w')]

[closed $((\mathrm{s}, \mathrm{x})]$

i.e. iff every contextually relevant situation whose temporal location is the night of the utterance, involving any Italian restaurant in all worlds which are just like ours, except for what is needed to allow for the existence of more Italian restaurants, and more relevant situations (and which is closest to a "no surprises" world) is a situation in which every Italian restaurant is closed.

\section{Summary}

The main claim I made in this paper is that besides the kind referring / quantificational genericity, originally introduced by Krifka 1987, natural language encodes an additional distinction between two kinds of modalized generic statement, both express a nonaccidental generalization. Both kinds of generalization are based on information we have on the actual world, but these are two different kinds of information, which lead to two different statements. The inductive ones are based on information about actual state of affairs, or the actual evidence we have, and state some "rule" or "pattern" true of actual world, i.e. assert that we should expect every such state of affairs to recur in other, close worlds as well. In contrast, the property oriented generalizations, are based on our knowledge or beliefs about some "rule" or pattern which is true of the actual world (the "in virtue of" property), and assert that this rule causally leads to another pattern (the one expressed by the predicate $)^{9}$.

I claimed that IS sentences can express property oriented generalizations only, whereas BP ones can express also inductive ones. I then formalized the two generalizations using two accessibility relations, and used them to account for a variety of semantic and distributional differences between BP and IS sentences in both habitual and temporally modified sentences. In addition I claimed that the similarity of BP sentences with sentences with proper names subjects suggests that the explanation for the IS/BP distinction lies in the distinction between referential and set expressions.

Several parts of this paper are still phrased in an intuitive and informal manner. In addition there are many open questions that the data and claims in the paper raise. In this sense there is still lots of work to be done in order to arrive at fully developed theory of the two kinds of genericity and the differences between IS and BP. Nonetheless, I believe the data and analysis presented here open a new and fruitful direction for the future understanding of genericity in natural language.

\section{Endnotes}


* I want to deeply thank Susan Rothstein for her continuous and invaluable professional and moral support in the various stages of working on this material, and Gennaro Chierchia and Angelika Kratzer for their time, kindness and the so many helpful comments and suggestions they gave me. Many thanks also to Maria Bittner, Greg Carlson, Veneeta Dayal, Daphne Heler, Manfred Krifka, Fred Landman, Orin Percus, Lenor Shoham, Adam Weiner and especially Ariel Cohen for their professional and other help.

${ }^{1}$ These sentences are fine under the existential reading of the IS subject. From now on I will relate only to the generic reading.

${ }^{2}$ The original observation of this distinction is due to Susan Rothstein (p.c.)

${ }^{3}$ Kamp's 1971 double indexing method for now seems appropriate for giving a fully formalized representation of this sentence.

${ }^{4}$ Brennan's 1993 distinctions between root and epistemic modals may help in explaining this data.

${ }^{5}$ The two accessibility relations associated with BP and IS sentences can also account for the "old intuition" about the more "definitional/normative" vs. "accidental" flavor of IS and BP sentences, respectively. But due to space limitations I cannot show that here. ${ }^{6}$ Recently, several people (like Chierchia 1996, Dobrovie-Sorin and Laca and Delfitto (1996) independently claimed that BP should be interpreted as kind referring even when combined with predicates of individuals.

${ }^{7}$ Gennaro Chierchia's comments (p.c.) helped me get at this schematic structure.

${ }^{8}$ This suggestion is due to Angelika Kratzer (p.c.). Delfitto 1996 has a similar suggestion.

${ }^{9}$ In this sense this distinction reminds of Kratzer's 1981 distinction between epistemic and circumstantial modality.

\section{References}

Brennan, V. (1993), "Root and Epistemic Modal Auxiliary Verbs", Ph.D. dissertation, Umass

Burton-Roberts, N. (1977), "Generic Sentences and Analyticity", Studies in Language 1. Carlson, G. (1977), "Reference to Kinds in English", Ph. D. dissertation, Umass.

Carlson, G. (1995), "Truth Conditions of Generic Sentences: Two Contrasting Views" in G. Carlson and F.J. Pelletier (eds.) The Generic Book, The University of Chicago Press, Chicago.

Chierchia, G. (1995) "Individual Level Predicates as Inherent Generics" in G. Carlson and F.J. Pelletier (eds.) The Generic Book, The University of Chicago Press, Chicago.

Chierchia, G. (1996), "Reference to Kinds Across Languages", ms. University of Milan.

Cohen, A. (1997) "Generics and Frequency Adverbs as Probability Judgments" in Proceedings of the Tenth Amsterdam Colloquium, Amsterdam, the Netherlands.

Condoravdi, C. (1992), "Strong and Weak Novelty and Familiarity" in Proceedings of SALT 2, Ohio State University Working Papers in Linguistics, 10, Ohio State University.

Declark, R. (1986), “The Manifold Interpretations of Generic sentences”, Lingua 68. Delfitto, D. (1997) “Aspect, Genericity and Bare Plurals” ms. Utrecht University. 
Dobrovie Sorin, C. and B. Laca (1996) "Generic Bare NPs”, ms. Universite Paris 7 and Universite de Strasbourg.

Kratzer, A. (1981), "The Notional Category of Modality" in H.-J. Eikmeyer \& H. Reiser (eds.) Worlds and Contexts. New Approaches to World Semantics. Berlin, de Gruyter.

Krifka, M. (1987), An outline of Genericity Partly in collaboration with C. Gerstner, SNS- Bericht, University of Tubingen.

Krifka M., Pelletier, F.J., Carlson G. ter Meulen A., Link, J. and G. Chierchia (1995). "Genericity: An Introduction" in G. Carlson and F.J. Pelletier (eds.) The Generic Book, The University of Chicago Press, Chicago.

Lewis, D. (1986) "Causation" in D. Lewis Philosophical Papers. Oxford University Press. Link, G. (1995) “Generic Information and Dependent Generics” in G. Carlson and F.J. Pelletier (eds.) The Generic Book, The University of Chicago Press, Chicago. de Swart, H. (1994) "(In)definites and Genericity", ms. University of Groningen. ter Meulen, A., (1995) "Semantic Constraints on Type Shifting Anaphora" in G. Carlson and F.J. Pelletier (eds.) The Generic Book, The University of Chicago Press, Chicago.

Wilkinson, C. (1991), "Studies in the Semantics of Generic Noun Phrases", Ph.D. dissertation, Umass, Amherst. 